\title{
Determinación de la concentración mínima y máxima del extracto de orégano (Origanum vulgare) como sustituto natural para preservar productos cárnicos
}

\author{
Ana Elizabeth Perlera de Escalante / José Miguel Bonilla Portillo / Franklin Kennedy Ventura Villegas / \\ Felipe Javier Alvarado Martínez \\ Facultad Multidisciplinaria de Ilobasco \\ Universidad Católica de El Salvador, El Salvador
}

Recepción: 02/10/2019 Aceptación: 05/05/2020

\section{Resumen}

En productos cárnicos crudos y cocidos suelen emplearse diferentes elementos para resaltar las características sensoriales (sabor, color), y alargar su vida útil.

La investigación comprendió la formulación y elaboración de chorizo mexicano y jamón tipo Virginia, utilizando diferentes dosis de aceite esencial de orégano; para comparar su efecto conservador frente a las sales de cura de nitrito de sodio. También se incluyó un control negativo por medio de pruebas microbiológicas en productos cárnicos elaborados. Se realizaron diez repeticiones de formulación para ambos productos en diferentes días.

De cada producción se tomaron muestras al azar para los análisis sensoriales y microbiológicos, encontrándose diferencias estadísticamente significativas, según prueba de Tukey $(\mathrm{p}<0.05)$; específicamente en las pruebas sensoriales para la muestra de jamón Virginia, con sales de cura de nitrito de sodio para los parámetros de color; y en la muestra de chorizo mexicano, con dosis de $50 \mathrm{ppm}$ de aceite esencial de orégano, respecto a parámetros de olor y preferencia.

Las pruebas microbiológicas para determinación de ausencia/presencia de Salmonella, y Listeria monocytogenes, E. coli, y coliformes, no mostraron diferencias estadísticamente significativas, según prueba de Tukey $(\mathrm{p}<0.05)$, para las muestras de jamón Virginia y para chorizo mexicano.

Palabras clave: Sales de nitrito, Salmonella, Listeria monocytogenes, E. coli, coliformes.

\begin{abstract}
In raw and cooked meat products, different elements are usually used to enhance the sensory characteristics (flavor, color), and extend their shelf life.

The research included the formulation and elaboration of Mexican chorizo and Virginia-type ham, using different doses of oregano essential oil; to compare its preservative effect against sodium nitrite cure salts. A negative control was also included, through microbiological tests on processed meat products. Ten repetitions of the formulation were carried out for both products on different days.

Random samples were taken from each production for sensory and microbiological analyzes, finding statistically significant differences, according to Tukey's test $(\mathrm{p}<0.05)$; specifically in the sensory tests for the Virginia ham sample, with sodium nitrite cure salts for the color parameters; and in the Mexican chorizo sample, with a dose of $50 \mathrm{ppm}$ of oregano essential oil, with respect to odor parameters and preference.

The microbiological tests to determine the absence / presence of Salmonella, and Listeria monocytogenes, E. coli, and coliforms, did not show statistically significant differences, according to the Tukey test $(\mathrm{p}<0.05)$, for the Virginia ham and Mexican chorizo samples.
\end{abstract}

Key words: Nitrite salts, Salmonella, Listeria monocytogenes, E. coli, coliforms.

1. Maestra en Horticultura, Docente tiempo completo; email: ana.perlera@catolica.edu.sv. ORCID: https://orcid.org/0000-0002-4353-8359

2. Maestro en Asesoría Educativa, Docente tiempo completo; email: jose.bonilla@catolica.edu.sv. ORCID: https://orcid.org/0000-0002-9559-9022

3. Técnico en Procesamiento de Alimentos, Docente tiempo completo; email: franklin.ventura@catolica.edu.sv. ORCID: https://orcid. org/0000-0003-3629-9289

4. Técnico en Procesamiento de Alimentos, Docente tiempo completo; email: felipe.alvarado@catolica.edu.sv. ORCID: https://orcid. org/0000-0002-7735-3125 


\section{Introducción}

El orégano (Origanum vulgare) se emplea en la elaboración de productos cárnicos como sustituto de nitritos, los cuales se utilizan en la industria alimentaria como inhibidores de microorganismos que pueden perjudicar la salud de los consumidores. Este componente pertenece a la familia Labiaceae, y es una planta herbácea vivaz muy aromática (Carhuapoma, 2006; Arcila-Lozano et al., 2004; D’Antuono et al., 2000). Sus hojas (tanto frescas como secas) se aprovechan como condimento en numerosas recetas culinarias por el excelente sabor que le confieren a las comidas (Albado et al., 2001).

Se ha demostrado que el orégano contiene aceites esenciales, por lo que no solo favorece a la salud humana, sino que además puede sustituir los aditivos sintéticos de los alimentos (Arcila-Lozano et al., 2004). El aceite esencial del orégano posee actividad antiradicalaria, cuya propiedad es atribuida a los mono fenoles carvacrol y timol, quienes son los principales quimiotipos. Cada uno de ellos tiene enzimas específicas que dirigen su biosíntesis (D'Antuono et al., 2000; Deighton et al., 1993).

La finalidad de los aceites esenciales es obtener compuestos aromáticos volátiles, producto del metabolismo de las plantas, cuyas características son las propiedades antimicrobianas. Gracias a esto, los aceites esenciales se han considerado como posibles sustitutos de los antibióticos en la alimentación animal (Hili et al., 1997). Dentro de este grupo de aceites con propiedades antimicrobianas destaca el aceite esencial de orégano.

De acuerdo con Burt (2004), el efecto antimicrobiano del orégano se debe, principalmente, a la presencia de metabolitos secundarios: carvacrol y timol $y$, en menor grado, $\gamma$-terpineno y p-cimeno. Estos compuestos dañan la integridad de la membrana celular de las bacterias, afectando la homeostasis y el equilibrio de los iones inorgánicos (Lambert et al., 2001).

Las tendencias actuales en el consumo de alimentos son de preferencia por alimentos más saludables, como los mínimamente procesados, reducidos en azúcar, edulcorantes alternativos naturales, y alimentos con tipos de formulación que declaren la menor cantidad de aditivos. Estos últimos son entendidos por el Diario Oficial de la comunidad Europea, como cualquier sustancia no consumida como alimento en sí; ni usado como ingrediente característico en la alimentación, independiente de que tenga valor nutritivo o no. También, que su adición intencionada a los productos alimenticios sea mediante un fin tecnológico en la fase de fabricación, transformación, preparación, tratamiento, envase, transporte y almacenamiento. Además, que tenga o pueda esperarse -razonable, directa o indirectamente- 
como resultado, que el propio aditivo o sus subproductos se conviertan en un componente de dichos productos alimenticios.

La seguridad alimentaria no solo hace referencia a la disponibilidad y acceso de alimentos para todas las personas, sino también a la calidad e inocuidad de estos. En este sentido, también los hábitos alimenticios de las personas están cambiando, y conduciendo a elegir alimentos cuyo contenido nutricional aporte a la salud sin representar un riesgo.

En la elaboración de productos cárnicos, tanto crudos como cocidos, en sus componentes destacan los retenedores que ayudan a su estabilidad, a su extensión y su actividad de agua; generando un efecto de sabor sobre el producto. Estos existen en distintas composiciones y suelen añadirse en forma de mezcla, según el resultado deseado en el producto terminado.

Los aditivos así empleados incluyen diferentes elementos que resaltan las características organolépticas: sabor, color, textura $y$, sobre todo, alargar su vida útil. Dentro de la formulación principal de algunos productos cárnicos cocidos se destacan los azúcares como depresores de la actividad del agua. Los azúcares de mezcla empleados para formular son diversos: van desde la sacarosa, dextrosa, lactosa, fructosa, los jarabes de glucosa obtenidos por hidrólisis de almidones hasta las dextrinas, que aportan atributos característicos en el aroma y sabor.
El producto terminado debe ser -en unidadun alimento cuyas características comerciales y de calidad e inocuidad sean adecuadas; de forma que su vida útil esté garantizada por conservadores permitidos.

La investigación mostró el comportamiento sensorial y de calidad encontrados, a través de la aplicación de los tratamientos con aceite de orégano (Origanum vulgare), como elemento de prueba en cuanto a sustituto de las sales de nitrito de sodio (sales de cura).

\section{Materiales y métodos}

La investigación se desarrolló en dos partes: la primera de ellas comprendió formulación y elaboración de los productos cárnicos; y la segunda, el análisis organoléptico y microbiológico de las muestras para comprobar la concentración de aceite de orégano recomendada, a fin de lograr la misma efectividad que causan las sales de nitrito utilizadas como preservante. Los porcentajes de aceite de orégano utilizados fueron establecidos con referencia a la concentración de nitritos que establece la Normativa Salvadoreña Obligatoria.

El proceso de elaboración y selección de formulaciones de prueba se realizó en los meses de mayo a julio. Posteriormente, en la planta de cárnicos de la Universidad Católica de El Salvador, Facultad Multidisciplinaria de Ilobasco-Cabañas, se procedió a las pruebas preliminares de compatibilidad de la fórmu- 
la para con los aceites esenciales de orégano: parámetros organolépticos de textura, características de las fibras de la mezcla cárnica, firmeza (es decir que el producto no se desmorone al contacto, y al mismo tiempo sea suave al tacto y al fraccionamiento al momento de masticarlo); el color y el olor que están determinados por el tipo de condimentos.

Las formulaciones iniciales comprendieron un grupo de cuatro cárnicos crudos y otro de cuatro cárnicos cocidos. Los productos seleccionados fueron: jamón tipo Virginia y chorizo mexicano. Las formulaciones de estos productos fueron dispuestas en dos grupos, con cinco tratamientos o formulaciones de diferente concentración de aceite esencial de orégano (una formulación de producto para el control negativo, y otra para el control positivo).

Los elementos que formaron parte de la formulación del jamón tipo Virginia fueron: carne de res, carne de cerdo, carne mecánica deshuesada mecánicamente $(\mathrm{CDM})^{5}$; soya, almidón de papa, harina de trigo, sales de cura (solo para el control negativo), aceite esencial de orégano (concentraciones de prueba), fosfato, azúcar, sal y condimentos.

De igual forma, para la formulación de chorizo mexicano se requirió de carne de res, carne de cerdo, CDM; grasa de cerdo, soya, almidón de papa, harina de trigo, sales de cura (solo para control negativo), aceite de orégano (concentraciones de prueba), fosfato, cebolla, ajo, jalapeño, pimiento y especias aromáticas.

Las actividades previas a este estudio comprendieron, además, una serie de diez producciones de los productos seleccionados; $\mathrm{y}$ el establecimiento de tres concentraciones de prueba del aceite de orégano adicionado. Esto se realizó tomando en cuenta los lineamientos industriales para el consumo oral del aceite de orégano, que recomiendan emplear otro aceite vegetal como vehículo para su utilización. De igual modo, con referencia a la Normativa Salvadoreña Obligatoria NSO 67.02.13:98, se establecieron tres concentraciones de trabajo comparables a las sales de cura, empleadas en la preservación de productos cárnicos. Durante las diez producciones se tomaron muestras al azar, tanto de jamón tipo Virginia como del chorizo mexicano; estas muestras estaban codificadas de acuerdo con la concentración de trabajo. (Ver tabla 1).

Las muestras fueron sometidas a análisis sensorial con enfoque en el producto descrito para la obtención del perfil sensorial, donde el panelista apreció, identificó y mesuró los atributos o características de una propiedad específica. El grupo de panelistas fue entrenado para identificar con precisión los atri- 
Tabla 1. Codificación de las muestras de productos cárnicos elaborados para la investigación

\begin{tabular}{|l|c|c|}
\hline \multirow{4}{*}{ Tipo de producto } & $\begin{array}{c}\text { Tratamiento aplicado al } \\
\text { producto }\end{array}$ & Código \\
\hline \multirow{5}{*}{ Jamón Virginia } & Aceite de oregano $50 \mathrm{ppm}$ & $\mathrm{J}-50$ \\
\cline { 2 - 3 } & Aceite de oregano $100 \mathrm{ppm}$ & $\mathrm{J}-100$ \\
\cline { 2 - 3 } & Aceite de oregano $150 \mathrm{ppm}$ & $\mathrm{J}-150$ \\
\cline { 2 - 3 } & Sal de cura $125 \mathrm{ppm}^{*}$ & $\mathrm{~J}-04$ \\
\cline { 2 - 3 } & No se aplicó conservante & $\mathrm{J}-05$ \\
\hline \multirow{5}{*}{ Chorizo mexicano } & Aceite de oregano $50 \mathrm{ppm}$ & $\mathrm{CH}-50$ \\
\hline & Aceite de oregano $100 \mathrm{ppm}$ & $\mathrm{CH}-100$ \\
\cline { 2 - 3 } & Aceite de oregano $150 \mathrm{ppm}$ & $\mathrm{CH}-150$ \\
\cline { 2 - 3 } & Sal de cura 125 ppm* & $\mathrm{CH}-04$ \\
\hline
\end{tabular}

Nota: ${ }^{\star S e ~ a p l i c o ́ ~ u n a ~ m u e s t r a ~ d e ~ r e f e r e n c i a . ~}$

butos en prueba; para ello se les solicitó: permanecer en ayuno mínimo de dos horas, no usar ningún producto de aseo personal con perfume y no consumir alcohol ni colorante durante los días que se realizaban las pruebas (Maqui Valdivia, J. L. 2017).

Para el análisis microbiológico, se realizaron pruebas para determinar la ausencia/presencia de bacterias como Salmonella, y Listeria monocytogenes; así como el análisis cuantitativo de E. coli, y coliformes, realizándose las pruebas por triplicado para cada muestra de jamón tipo Virginia y para chorizo mexicano.
Los datos obtenidos del análisis cuantitativo microbiológico y sensorial se sometieron a una prueba análisis de varianza (ANOVA). La comparación de medias fue hecha con la prueba de Tukey, aplicando el nivel de confiabilidad de $\mathrm{P}<0.05$, usando el software estadístico Infostat 2018.

\section{Resultados y discusión}

Anteriormente, el análisis organoléptico o llamado también sensorial era utilizado como un método marginal para evaluar la calidad en los alimentos. Pero, actualmente, esta he- 
rramienta permite una forma muy útil de conocer la aceptación del consumidor con respecto a un determinado producto.

\section{Formulación y análisis organoléptico del jamón familiar}

Se seleccionaron dos formulaciones de productos cárnicos: la primera, correspondió al jamón tipo Virginia; y la otra, a chorizo mexicano. La primera formulación establecida se reprodujo simultáneamente para cinco concentraciones utilizadas como tratamientos, que incluían tres concentraciones de aceite de orégano (50 ppm, 100 ppm, 150 ppm); una de 125 ppm de sales de cura, y un tratamiento de 0 ppm de sales de nitrito y aceite de orégano.

El jamón tipo Virginia elaborado en los tratamientos de aceite de orégano y su referente sales de cura, fueron sometidos a consentimiento de sus características organolépticas para establecer las diferencias y aceptación del producto. La herramienta de prueba para las características organolépticas mencionadas fue una encuesta, donde se seleccionó una sola respuesta.

Los resultados obtenidos durante el período de estudio mostraron los atributos más observables en las formulaciones que contenían aceite de orégano, comparado a la formulación que contenía sales de cura (conocida como muestra de referencia).
Dentro de las primeras características observadas por el panel de análisis sensorial, los evaluadores coincidieron en que, dentro de ninguno de los tratamientos realizados con aceite de orégano aplicado ( $<-50, \mathrm{~J}-100$ y J-150) se encontró el color rosa pálido característico, que sí otorga a los productos cárnicos la sal de cura (muestra de referencia). Esto representa una diferencia significativa para la muestra de referencia ( $\mathrm{p}=0.0011$ ), cuya formulación contiene sales de cura (Tabla 2.).

$\mathrm{Al}$ evaluar las características sensoriales del olor y sabor, dos de las muestras de jamón elaborado con aceite de orégano (50 ppm y 100 ppm) y la muestra hecha con sales de cura (muestra de referencia) fueron las mejor aceptadas. De entre las muestras de aceite de orégano, la concentración de mayor aprobación fue J-100.

En el momento de ofrecer las diferentes muestras de jamón a los panelistas, estas se prepararon de tal forma que fueran recientes, cuidando que la apariencia no mostrara rasgos de deterioro o alteración. Se observó que el jamón que no contenía conservadores resaltó aún más el sabor a condimentos y a la mezcla de carnes, a diferencia de las otras. Este aspecto marcó con más naturalidad el sabor e influyó en la aceptación y preferencia de estas muestras por encima del resto. 
Tabla 2. Perfil descriptivo del análisis sensorial empleadas para el jamón tipo Virginia, empleando diferentes tratamientos para su preservación

\begin{tabular}{|l|c|c|c|c|}
\hline \multicolumn{1}{|c|}{ Tratamiento } & $\begin{array}{c}\text { Color } \\
\text { similar a la } \\
\text { muestra** }\end{array}$ & $\begin{array}{c}\text { Olor similar } \\
\mathbf{a} \text { la } \\
\text { muestra** }\end{array}$ & $\begin{array}{c}\text { Sabor similar } \\
\mathbf{a} \text { la } \\
\text { muestra** }^{*}\end{array}$ & Preferencia** $^{*}$ \\
\hline $\begin{array}{l}\text { Jamón tipo Virginia }+ \\
\text { aceite orégano } 50 \mathrm{ppm}(\mathrm{J} \\
50)\end{array}$ & $1.1 \pm 1.7 \mathrm{a}$ & $2.3 \pm 1.6 \mathrm{a}$ & $2.6 \pm 1.8 \mathrm{a}$ & $2.9 \pm 1.8 \mathrm{~b}$ \\
\hline $\begin{array}{l}\text { Jamón tipo Virginia }+ \\
\text { aceite orégano } 100 \mathrm{ppm}(\mathrm{J} \\
\text { 100) }\end{array}$ & $1.7 \pm 3.0 \mathrm{a}$ & $3.4 \pm 1.9 \mathrm{a}$ & $3.5 \pm 2.1 \mathrm{a}$ & $3.4 \pm 1.9 \mathrm{~b}$ \\
\hline $\begin{array}{l}\text { Jamón tipo Virginia }+ \\
\text { aceite orégano } 150 \mathrm{ppm}(\mathrm{J} \\
\text { 150) }\end{array}$ & $1.1 \pm 2.1 \mathrm{a}$ & $1.6 \pm 1.5 \mathrm{a}$ & $2.1 \pm 1.6 \mathrm{a}$ & $1.3 \pm 1.5 \mathrm{ab}$ \\
\hline $\begin{array}{l}\text { Jamón tipo Virginia }+ \text { sal } \\
\text { de cura 125 ppm (J 04) }\end{array}$ & $6.2 \pm 4.4 \mathrm{~b}$ & $2.7 \pm 2.2 \mathrm{a}$ & $1.8 \pm 1.7 \mathrm{a}$ & $2.1 \pm 1.4 \mathrm{ab}$ \\
\hline $\begin{array}{l}\text { Jamón Virginia sin ningún } \\
\text { tratamiento. J-05 }\end{array}$ & & & & $0.3 \pm 0.5 \mathrm{a}$ \\
\hline
\end{tabular}

Nota: ${ }^{\star}$ Se aplicó muestra de referencia. ${ }^{*}$ Dentro de cada columna, los valores poseen una letra en común. No son significativamente diferentes entre sí, según la prueba de Tukey p $<0.05$.

\section{Formulación y análisis organoléptico de chorizo mexicano}

La elaboración de chorizo mexicano, comprendió una formulación estándar, difiriendo únicamente en la sal de cura de nitrito de sodio agregada para guardar la inocuidad de la muestra y por la particularidad de proporcionar un color rosa en los productos cárnicos, en esta parte de la formulación se separan las mezclas cárnicas para agregar a una de ellas un sustituto de nitrito de sodio y a la otra parte de la mezcla cárnica el aceite esencial de orégano. Esta variante en la formulación fue observada por el grupo de panelistas como parte de las pruebas organolépticas, estableciendo entre sus comentarios respecto a cada formulación elaborada con diferentes concentraciones de aceite esencial de orégano y la concentración estándar para nitrito de sodio, si existía un color diferente entre ellas, sobre todo por haber utilizado diferentes porcentajes de aceite esencial de orégano.

De los productos elaborados con las diferentes concentraciones de aceite esencial de orégano a 50 ppm, 100 ppm y 150 ppm, fue mejor aceptada la de menor concentración, es decir la de 50 ppm de aceite de orégano. En algunas ocasiones también se ha logrado determinar cómo el tipo de preparación de un producto cárnico puede modificar las propiedades sensoriales, debido a la estructura de las proteínas aportada por el tipo de mezcla cárnica (Mahecha, H.S. y otros, 2011). 
Las pruebas sensoriales de aroma para el chorizo mexicano, tanto con aceite esencial de orégano como el elaborado con nitrito de sodio, sólo mostró los atributos percibidos a partir de las especies, condimentos y la carne de res y cerdo en conjunto. Las muestras no fueron discriminadas por poseer el sustituto de conservador en prueba, para este caso el aceite esencial de orégano. El aroma fue igualmente apreciado para las diferentes muestras de chorizo.

En las formulaciones de productos cárnicos, es importante conservar las características sensoriales y también las microbiológicas, para ello deben adecuarse las concentraciones de aditivo de forma sinérgica con los demás ingredientes, cuidando los límites permitidos por los organismos reguladores. Los aditivos de origen químico pueden potenciar el sabor y mejorar el color en los embutidos, al usarse aceite de orégano en concentraciones bajas, permitió que el color natural de los productos elaborados no fuera modificado y mantener un color semejante a los que poseen sales de cura (ver tabla 3 ).

\section{Análisis microbiológico y físicos del ja- món familiar}

Los análisis microbiológicos fueron realizados para determinar la presencia de Listeria monocytogenes, Salmonella, E. coli y coliformes. De acuerdo con los resultados, la presencia de los dos primeros patógenos en las muestras fue negativa (ver figura 1).

Por su parte, el análisis de E. coli y coliformes totales se realizó para todas las muestras por igual (figura 1), tanto en los productos elaborados con aceite esencial de orégano a diferentes concentraciones,

Tabla 3. Perfil descriptivo del análisis sensorial empleado para el chorizo mexicano, en diferentes tratamientos para su preservación

\begin{tabular}{|l|c|c|c|c|}
\hline \multicolumn{1}{|c|}{ Tratamiento } & $\begin{array}{c}\text { Color } \\
\text { similar a la } \\
\text { muestra* }\end{array}$ & $\begin{array}{c}\text { Olor } \\
\text { similar a la } \\
\text { muestra* }\end{array}$ & $\begin{array}{c}\text { Sabor } \\
\text { similar a la } \\
\text { muestra* }\end{array}$ & Preferencia* \\
\hline $\begin{array}{l}\text { Chorizo mexicano + aceite } \\
\text { orégano 50 ppm (CH-50) }\end{array}$ & $2.6 \pm 1.9 \mathrm{a}$ & $4.2 \pm 2.3 \mathrm{c}$ & $3.8 \pm 1.8 \mathrm{~b}$ & $4.3 \pm 2.2 \mathrm{c}$ \\
\hline $\begin{array}{l}\text { Chorizo mexicano + aceite } \\
\text { orégano 100 ppm (CH-100) }\end{array}$ & $2.2 \pm 2.3 \mathrm{a}$ & $3.4 \pm 1.9 \mathrm{bc}$ & $3.4 \pm 1.6 \mathrm{ab}$ & $3.0 \pm 1.7 \mathrm{bc}$ \\
\hline $\begin{array}{l}\text { Chorizo mexicano + aceite } \\
\text { orégano 150 ppm (CH-150) }\end{array}$ & $2.3 \pm 2.0 \mathrm{a}$ & $1.9 \pm 1.7 \mathrm{ab}$ & $1.60 \pm 1.5 \mathrm{a}$ & $1.0 \pm 1.3 \mathrm{ab}$ \\
\hline $\begin{array}{l}\text { Chorizo mexicano + sal de cura } \\
\text { 125 ppm (CH-04) }\end{array}$ & $3.3 \pm 3.2 \mathrm{a}$ & $1.1 \pm 1.1 \mathrm{a}$ & $1.8 \pm 2.4 \mathrm{ab}$ & $1.9 \pm 2.2 \mathrm{ab}$ \\
\hline $\begin{array}{l}\text { Chorizo sin ningún tratamiento } \\
\text { (CH-05) }\end{array}$ & & & & \\
\hline
\end{tabular}

Nota: ${ }^{*}$ Dentro de cada columna, los valores que poseen una letra en común no son significativamente diferentes entre sí, según la prueba de Tukey $\mathrm{p}<0.05$. 


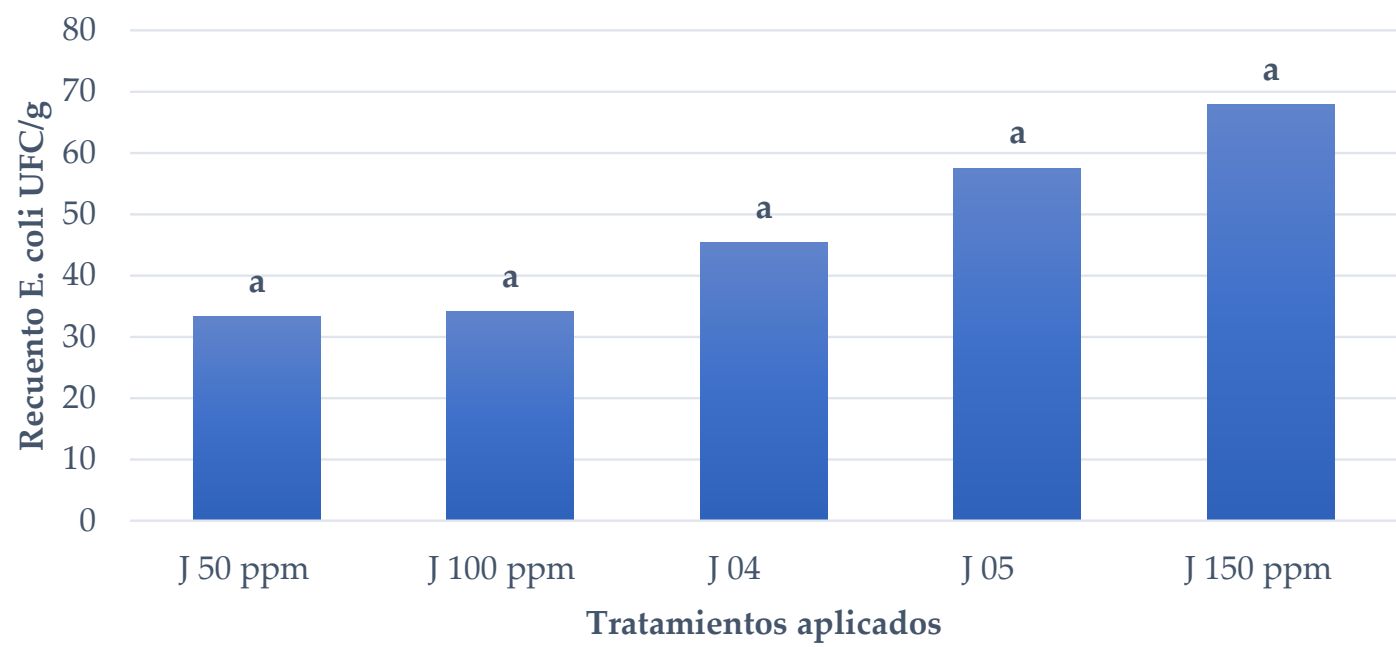

Figura 1. Medias para el recuento de E. coli, en jamón Virginia utilizando los distintos tratamientos. (Aunque posean la misma letra, no son estadísticamente significativas según la prueba de Tukey $\mathrm{p}<0.05$ ).

como en los elaborados con sales de nitrito de sodio. Esto con la intención de observar la inhibición de bacterias.

El análisis microbiológico para recuento de coliformes permite saber cuán posible es la contaminación de la muestra, durante el proceso de elaboración de los productos cárnicos, respecto al manejo por parte de los manipuladores. Es importante realizar un conteo total de coliformes, debido a la posibilidad de encontrar bacterias como E. coli.

En muchos casos, la manipulación de alimentos puede mostrar resultados positivos de la presencia de E. coli. Esta bacteria tiene distintas cepas que, en su mayoría, no causan daño; a excepción de la que produce la verotoxina (E. coli O157:H7)(Zamora, J., y otros 2010) (González, A. P., 2018). Dentro de la investigación, los resultados reportaron la presencia cepas de E. coli en un $30 \%$ de las muestras estudiadas, pero ninguna de ellas era la toxigénica.

Uno de los elementos más importantes en la investigación fue conocer el potencial conservador presentado por el aceite esencial de orégano en productos cárnicos; así como comparar la inhibición de bacterias patógenas en el alimento, respecto a las sales de nitrito de sodio. En cuanto a esto, se mostraron recuentos bajos de coliformes totales para cárnicos con dosis de 50 ppm de aceite esencial de orégano, a semejanza de los cárnicos con nitrito de sodio. (Ver figura 2).

El efecto antimicrobiano de las sales de cura es debido al ion $\mathrm{NO}_{2}$ que, en condiciones de $\mathrm{pH}$ bajo, inhibe el crecimiento de microorga- 


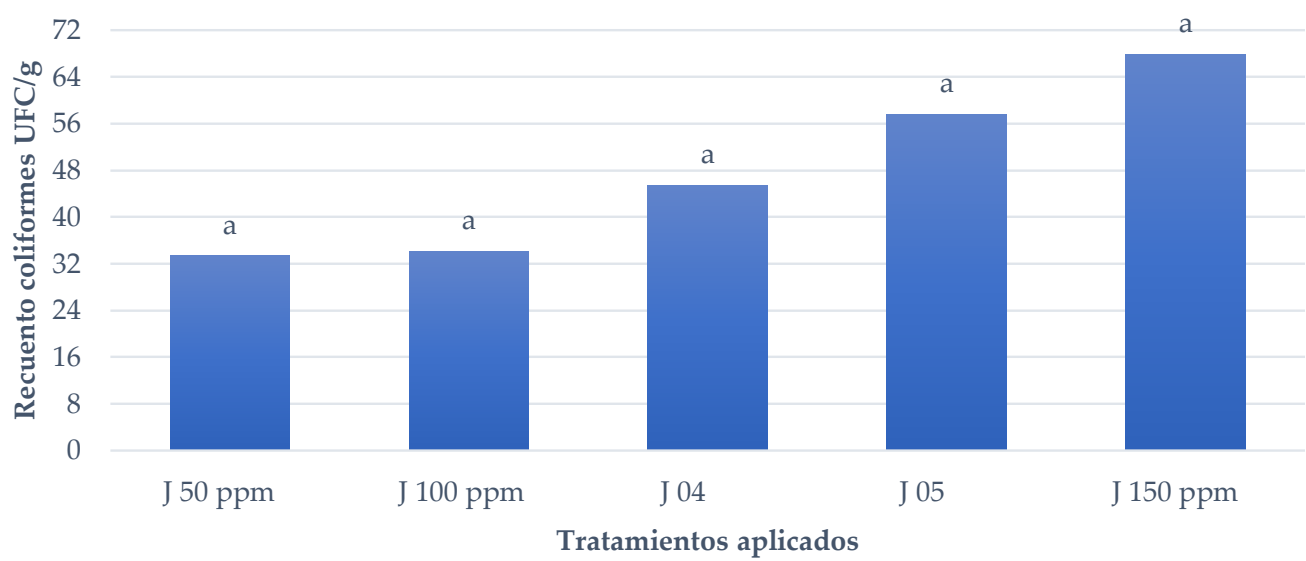

Figura 2. Medias para el recuento de coliformes en jamón Virginia en los diversos tratamientos. (Aunque posean la misma letra, no son estadísticamente significativas según la prueba de Tukey $\mathrm{p}<0.05)$.

nismos y retarda la oxidación de los ácidos grasos insaturados, presentes en los ingredientes de la formulación del producto (Lugo, E. 2008) (Zurbriggen, C. 2009).

Una de las condiciones idóneas para el crecimiento de microorganismos es el contenido de agua en el alimento. Para el caso de la investigación, los porcentajes de humedad reportados en el análisis físico del jamón Virginia fueron más altos que los establecidos por la NSO 67.02.13:98 (tabla 4).

\section{Análisis microbiológico y físico de chori- zo mexicano}

El recuento de E. coli en las formulaciones de chorizo mexicano mostró valores inferiores a los del jamón (figura 3). Según la formulación del chorizo mexicano, la utilización de cilantro (Coriandrum sativum) y pimiento verde (Capsicum annum), así como de los aceites de tomillo, romero y apio favorecen la conservación del alimento (Calderón Sánchez, B. 2019). Esto se considera un efecto coadyuvante, respecto a los tratamientos en donde se utilizó aceite de orégano (ver figura 3).

Por su parte, el recuento total para coliformes presentó valores menores a $100 \mathrm{UFC/g}$, cumpliendo con los parámetros microbiológicos de la NSO de productos cárnicos (figura 4). Estos valores se compararon con los de la figura 3, al mostrar valores superiores a > $10 \mathrm{UFC/g}$ y menor a $100 \mathrm{UFC/g}$, para la presencia de E. coli; aunque no se trataba de la cepa patógena O157:H7. La normativa Salvadoreña Obligatoria recomienda para la elaboración de este tipo de alimentos, implementar controles bajo buenas prácticas de manufactura en la manipulación del alimento (ver figura 4 ). 
Tabla 4. Contenido de humedad analizado en el jamón Virginia.

\begin{tabular}{|l|c|c|}
\hline \multicolumn{1}{|c|}{ Tratamiento } & $\begin{array}{c}\text { Porcentaje de } \\
\text { Humedad }^{*}\end{array}$ & $\begin{array}{c}\text { Peso en gramos por unidad en } \\
\text { rodaja de jamón Virginia* }\end{array}$ \\
\hline $\begin{array}{l}\text { Jamón + aceite orégano } \\
\text { 50ppm (J 50 ppm) }\end{array}$ & $67.93 \mathrm{a}$ & $6.87 \mathrm{a}$ \\
\hline $\begin{array}{l}\text { Jamón + aceite orégano } \\
\text { 100ppm (J 100 ppm) }\end{array}$ & $67.19 \mathrm{a}$ & $6.67 \mathrm{a}$ \\
\hline $\begin{array}{l}\text { Jamón + aceite orégano } \\
\text { 150ppm (J 150 ppm) }\end{array}$ & $66.57 \mathrm{a}$ & $6.41 \mathrm{a}$ \\
\hline $\begin{array}{l}\text { Jamón + sales de nitrito } \\
125 \text { ppm ( J 04) }\end{array}$ & $67.3 \mathrm{a}$ & $6.69 \mathrm{a}$ \\
\hline $\begin{array}{l}\text { Jamón sin aceite orégano / sales de } \\
\text { nitrito (J 05) }\end{array}$ & $67.95 \mathrm{a}$ & $6.75 \mathrm{a}$ \\
\hline
\end{tabular}

Nota: ${ }^{\star}$ Dentro de cada columna, los valores que poseen una letra común no son significativamente diferentes entre sí, según la prueba de Tukey $\mathrm{p}<0.05$.

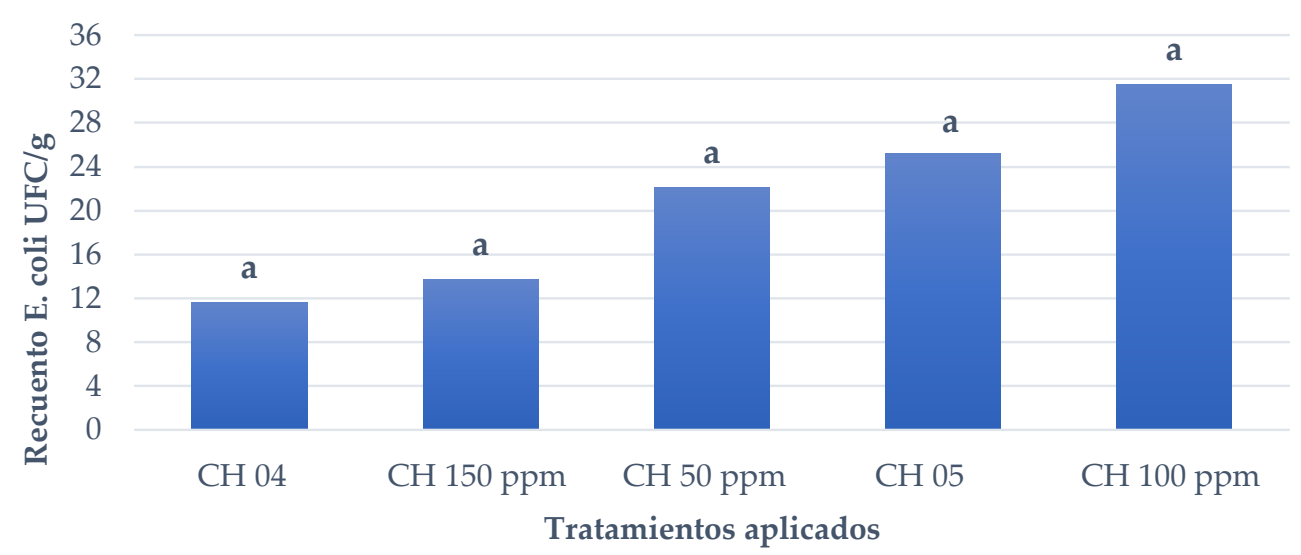

Figura 3. Medias para el recuento de E. coli en chorizo mexicano en los diversos tratamientos. (Aunque posean la misma letra, no son estadísticamente significativas según la prueba de Tukey $\mathrm{p}<0.05)$.

La calidad de un alimento está determinada principalmente por su inocuidad y calidad. Si bien es cierto que, en términos de calidad, puede conducirse a selección de materias primas de mejor o menor calidad para dar a los productos atributos sensoriales favorables (olor, sabor y color característico, como en el caso de los nitritos), que aporten significativamente en el desarrollo de color y sabor (Lugo, E. B. 2008) (García, E. M. y otros 2018).

Sin embargo, la inocuidad no puede ofrecerse en términos de menor o mejor, como podría pasar con cualidades de productos que se co- 


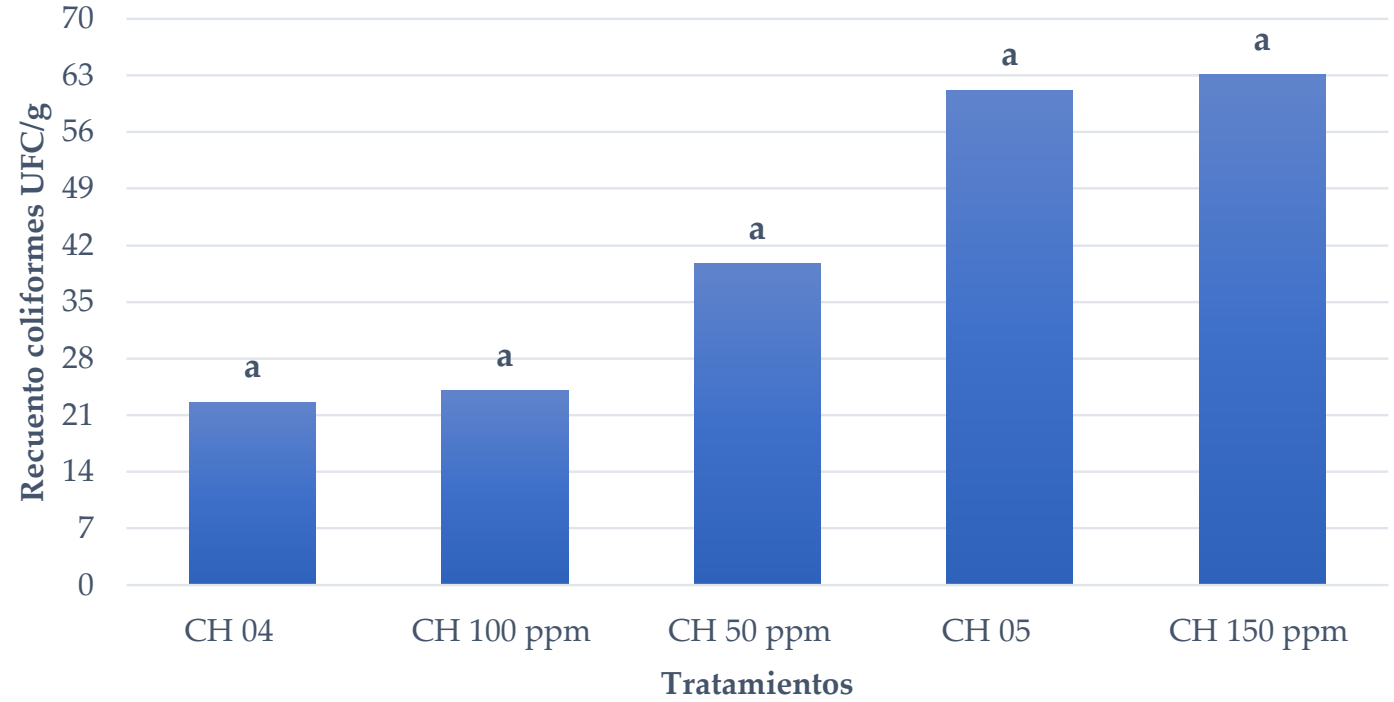

Figura 4. Medias para el recuento de coliformes en chorizo mexicano en los diversos tratamientos. (Aunque posean la misma letra, no son estadísticamente significativas según la prueba de Tukey $\mathrm{p}<0.05)$.

mercializan de acuerdo a la materia prima con la que se elaboran. Por este motivo, evitar la contaminación y proliferación de microorganismos es crucial, debido a que los análisis de humedad permiten conocer la presencia de la misma dentro del producto; y cómo esta puede afectar la vida útil de anaquel.

En el análisis realizado al chorizo mexicano, se determinó una humedad homogénea para todas las muestras analizadas. Las medias reflejan que todas ellas están dentro de los parámetros exigidos por la NSO (Normas Salvadoreñas Obligatorias) para cárnicos, sin mostrar diferencias significativas entre los tratamientos aplicados (ver tabla 5).

\section{Conclusiones}

Las formulaciones de los productos cárnicos elaborados con aceite esencial de orégano (jamón Virginia y chorizo mexicano) fueron sometidas a análisis microbiológico, para conocer el efecto conservador de este, respecto a las propiedades de las sales de cura de nitrito de sodio, las cuales son comúnmente utilizadas para este fin.

El aceite esencial de orégano se incorporó a las formulaciones en concentraciones del 50, 100 y 150 ppm, notándose mejor aceptación sensorial para el jamón con aceite de orégano a 100 ppm y para el chorizo mexicano a 50 ppm. Los resultados microbiológicos mostraron mayor número de colonias (UFC/g) de $E$. coli para el jamón en relación al chorizo mexicano; esto debido al aporte conservador que 
Tabla 5. Resultados de análisis físico de porcentaje de humedad y peso promedio en chorizo mexicano

\begin{tabular}{|l|c|c|}
\hline \multicolumn{1}{|c|}{ Tratamiento } & Humedad* & $\begin{array}{c}\text { Peso en gramos por } \\
\text { unidad de chorizo } \\
\text { mexicano* }\end{array}$ \\
\hline $\begin{array}{l}\text { Chorizo + aceite de orégano 50 } \\
\text { ppm (CH 50 ppm) }\end{array}$ & $61.57 \mathrm{a}$ & $18.96 \mathrm{a}$ \\
\hline $\begin{array}{l}\text { Chorizo + aceite de orégano 100 } \\
\text { ppm (CH 100 ppm) }\end{array}$ & $60.23 \mathrm{a}$ & $17.78 \mathrm{a}$ \\
\hline $\begin{array}{l}\text { Chorizo + aceite de orégano 150 } \\
\text { ppm (CH 150 ppm) }\end{array}$ & $62.70 \mathrm{a}$ & $18.86 \mathrm{a}$ \\
\hline $\begin{array}{l}\text { Chorizo + sales de nitrito 125 } \\
\text { ppm (CH 04) }\end{array}$ & $62.23 \mathrm{a}$ & $19.94 \mathrm{a}$ \\
\hline $\begin{array}{l}\text { Chorizo sin aceite de orégano / } \\
\text { sales de nitrito (CH 05) }\end{array}$ & $63.89 \mathrm{a}$ & \\
\hline
\end{tabular}

Nota: ${ }^{\star}$ Dentro de cada columna, los valores que poseen una letra en común no son significativamente diferentes entre sí, según la prueba de Tukey $\mathrm{p}<0.05$.

brindan las especias y vegetales incorporados al producto. Como resultado, la adhesión de estos elementos junto con las concentraciones de aceite de orégano utilizadas, mejoran la vida útil del producto.

El aceite esencial de orégano demostró mejor dosis de aplicación para las formulaciones cárnicas a concentraciones de 50 y 100 partes por millón para el jamón Virginia; y en concentración de 150 partes por millón para el chorizo mexicano.

El efecto conservador del aceite esencial de orégano aplicado para jamón y chorizo mexicano, en las concentraciones de 50 y 100 ppm, mostró cualidades sensoriales de sabor, color, olor y textura propias del producto hasta el día 20 de almacenamiento; conservando el producto a una temperatura de $4^{\circ} \mathrm{C}$.
Estos atributos sensoriales están en concordancia con la conservación de los productos formulados, coincidiendo con estudios en donde se ha comprobado la eficacia antimicrobiana de los fitoquímicos contenidos en el aceite esencial, los cuales inhiben el crecimiento de bacterias patógenas que pueden colonizar un alimento, como Salmonella spp., y E. coli.

Entre los componentes que favorecen la inhibición del crecimiento microbiano se encuentra el carvacrol, el cual se encontraba en mayor proporción dentro del aceite esencial de orégano, que se utilizó en las formulaciones cárnicas como sustituto de las sales de cura de nitrito de sodio (Dorman, H. and Deans, S., 2000; Pundir, R., and Sharma, C., 2010). 


\section{Referencias}

Acevedo, D.; Navarro, M. y Monroy, L. (2013). Composición química del aceite esencial de hojas de orégano (Origanum vulgare). Información tecnológica, 24(4), 43-48.

Arango B., O.; Pantoja D., D; Santacruz Ch., L. y Hurtado B., A. M.(2012). Actividad antioxidante del aceite esencial de orégano (Lippia origanoides H. B. K) del Alto Patia. Biotecnología en el Sector Agropecuario y Agroindustrial, 10 (2), 79-86.

Bazán-Lugo, E. (2008). Nitritos y Nitratos: Su uso, control y alternativas en embutidos cárnicos. Nacameh, 2 (2), 160-187. Recuperado de https://dialnet.unirioja.es/servlet/articulo? codigo $=3664829$

Calderón-Sánchez, B. S. y Giler-Pín, Y. E. (2019). Uso de vegetales como sustitutos de conservantes en la elaboración de embutidos (chorizo) (Tesis de pregrado). Facultad de Ingeniería Química, Universidad de Guayaquil, Educador.

Dorman, H. y Deans, S. (2000). Antimicrobial agents from plants: antibacterial activity of plant volatile oils. Journal of applied microbiology, (88), 308-316. Recuperado de https://sfamjournals.onlinelibrary.wiley.com/doi/full/10.1046/j.1365-2672.2000.00969.x

Gallego R., J. A. (2014). Fuente alternativa de nitratos para la industria cárnica: Influencia del extracto de apio y cultivos iniciadores sobre el color del jamón cocido tipo Medellín (Tesis doctoral), Departamento de Ingeniería, Escuela Politécnica Superior de Orihuela, Universidad Miguel Hernández, España.

Gámez-Piñón, J. R.; Rentería-Monterrubio, A. L.; Durán-Meléndez, L. A.; Chávez-Martínez, A.; Alarcón-Rojo, A. D.; Aguilar-Palma, N.G. y Silva-Vásquez, R. (2015). Efecto del aceite esencial de orégano en el rendimiento y las propiedades fisicoquímicas y microbiológicas de la carne de pollo. Investigación y Ciencia, 23 (66), 5-11.

González-Antonio, P. (2018). Identificación de los genes: stx1, stx2, eaea y hlya, en cepas de Escherichia coli aisladas de canales y carne procesada de ovinos (Tesis de pregrado). Facultad de Medicina Veterinaria y Zootecnica, Universidad Autónoma del Estado de México, Toluca, México. Recuperado de http://ri.uaemex.mx/handle/20.500.11799/94389 
Hernández-García, E. M.; González-de-la-Cruz, J. U.; de-la-Cruz-Leyva, M.C.; Pérez-Sánchez, C. del C.; Guzmán-Ceferino, J.; Ramírez-Muñoz, I.Y. y Durán-Mendoza, T. (2018). Hibiscus sabdariffa L. en un embutido cárnico y su efecto en las características fisicoquímicas, nutritivas, microbiológicas, y aceptación sensorial. Nacameh, 12(2), 15-29. Recuperado de https://dialnet.unirioja.es/servlet/articulo? codigo=7006666

Kumar-Pundir, R.; Jain, P. y Sharma, C. (2010). Antimicrobial Activity of Ethanolic Extracts of Syzygium aromaticum and Allium sativum Against Food Associated Bacteria and Fungi. Ethnobotanical Leaflets, 14 (3)

Maqui-Valdivia, J.L. (2017). Evaluación de la influencia del aceite esencial de orégano (Origanum vulgare) y pasta de ajo (Allium Sativum) sobre la estabilidad fisicoquímica y sensorial del chorizo de cerdo (Tesis de pregrado). Escuela Profesional de Ingeniería Química, Facultad de Ingeniería, Universidad Nacional Jorge Basadre Grohmann-Tacna, Perú.

Méndez-Zamora, G.;Durán-Meléndez, L. A.; Aquino-López, J. L.; Santellano-Estrada, E. y Silva-Vázquez, R. (2016). Efecto del aceite de orégano (Poliomintha longiflora Gray) sobre la productividad y calidad de carne de conejos. Ecosistemas y recursos agropecuarios, $3(8), 259-265$.

Méndez-Zamora, G.; García-Macías, J. A.; Durán-Meléndez, L. A.; Herman-Lara, E.; Santellano-Estrada, E. y Silva-Vázquez, R. (2015). Aceite esencial de orégano (Lippia berlandieri Schauer) en variables de calidad de la canal de pollo. Ecosistemas y recursos agropecuarios, 2 (4), 41-51.

Menéndez-Zamora, G.; García-Macías, J.A.; Santella-Estrada, E.; Durán-Meléndez, L. A. y Silva-Vázquez, R. (2015). Aceite de orégano sobre la calidad de pechuga de pollos de engorda. Investigación y Ciencia, 23 (65), 5-12

Montiel-Flores, E.; López-Malo, A. y Bárcenas-Pozos, M. E. (2013). Vegetales como fuentes de nitritos: una alternativa para el curado de carnes. Temas Selectos de Ingeniería de Alimentos, 7 (1), 57-67.

Suárez-Mahecha, H.; Restrepo-Molina, D.A. y Carrasquilla-Galeano, L. A. (2011). Influencia de especias naturales en la vida útil y aceptación sensorial de salchicha Bratwurst. Revista Facultad Nacional de Agronomía Medellín, 64(1), 6007-6013. 
Zamora, J.; Reinhardt, G.; Polette, M. y Macías, P. (2000). Detección rápida en el diagnóstico de Escherichia coli toxigénica productora de LT y VT. Archivos de medicina veterinaria, 32 (1), 83-87. Recuperado de https://dx.doi.org/10.4067/S0301-732X2000000100010

Zurbriggen, C. J. (2009). Comparación de los diversos factores que influyen sobre el desarrollo del color en las distintas etapas de elaboración de pastas de productos cárnicos crudo-curados (Tesis de maestría). Facultad de Ciencias Veterinarias, Universidad Nacional del Litoral, Argentina. Recuperado de https://bibliotecavirtual.unl.edu.ar:8443/bitstream/handle/11185/265/tesis.pdf? sequence=1\&isAllowed $=y$ 